\title{
Obtención de la primera variedad argentina de mostaza india: SANTA CATALINA UNLP, para su utilización como biofumigante
}

\author{
Perniola, Omar Salvador 1,4; Chorzempa, Silvia Elena ; D'Amico, Marco ${ }^{1,2,3}$; Staltari, Sebastián'; \\ Molina, María del Carmen ${ }^{1}$; Astiz Gassó, Marta Mónica ${ }^{1}$ \\ ${ }^{1}$ Instituto Fitotécnico de Santa Catalina, Facultad de Ciencias Agrarias y Forestales, Universidad \\ Nacional de La Plata, ${ }^{2}$ Facultad de Ciencias Agrarias, Universidad Nacional de Lomas de \\ Zamora, ${ }^{3}$ Estación Experimental Gorina, Ministerio de Desarrollo Agrario de la Provincia de \\ Buenos Aires, ${ }^{4}$ omarperniola@yahoo.com.ar
}

Resumen. En las últimas décadas, la mayor concientización de la sociedad sobre los efectos nocivos de los agroquímicos sobre la salud y el medio ambiente, ha generado cambios en algunos establecimientos hortícolas y florícolas del cinturón verde de Buenos Aires, que han incorporado otras estrategias de control de plagas, amigables con el medio ambiente, con la consecuente reducción en el uso de plaguicidas sintéticos. En este contexto, la biofumigación con mostaza india (Brassica juncea L. Czerniak) se presenta como una estrategia biológica eficaz para el control de plagas de suelo, que podría formar parte de un programa de manejo agroecológico de plagas. En nuestro país, hay registros de prácticas de biofumigación con recursos locales (principalmente, restos de cosecha de especies hortícolas brasicáceas), pero no existían desarrollos de cultivares con propósitos biofumigantes. Por este motivo, se realizó un proceso de selección masal y se obtuvo la variedad biofumigante SANTA CATALINA UNLP, que es el primer cultivar de mostaza india inscripto en el Registro Nacional de Cultivares del INASE.

La variedad de mostaza india SANTA CATALINA UNLP fue desarrollada para su utilización como biofumigante de suelos y sustratos en producciones hortícolas y florícolas. Dado que el proceso de selección se realizó en la localidad de Llavallol, conurbano bonaerense, este cultivar se adapta a las condiciones medioambientales del cinturón verde de Buenos Aires. Numerosas investigaciones realizadas durante el período de selección del cultivar, han 
Innovación y Desarrollo Tecnológico y Social (2021) 3 (1): 1- 23

demostrado que la biofumigación con esta variedad es eficaz para suprimir algunas especies de hongos fitopatógenos, reducir poblaciones de nematodos fitófagos y para inhibir la emergencia y el crecimiento de algunas malezas. También se demostró que la biofumigación con mostaza india no afecta al hongo antagonista Trichoderma spp. ni a los nematodos de vida libre del suelo (benéficos).

La variedad SANTA CATALINA UNLP fue desarrollada en el Instituto Fitotécnico de Santa Catalina, dependencia de la Facultad de Ciencias Agrarias y Forestales de la Universidad Nacional de La Plata. Desde el año 2009 hasta el 2016 se realizó selección masal sobre una población mixta proveniente de un lote de semillas de mostaza india que ingresó al país procedente de la India, para ser comercializado como condimento. Se seleccionaron las plantas con mayor biomasa aérea y con menor incidencia y severidad de podredumbre negra o mancha en $\mathrm{V}$ (Xanthomonas campestris pv. campestris). Paralelamente se realizaron ensayos para evaluar su capacidad como biofumigante para el biocontrol de plagas agrícolas. En junio de 2017 fue multiplicada, verificándose la estabilidad de la variedad. En varios ensayos se observó que la biofumigación con mostaza india tuvo efecto supresor sobre el crecimiento de Fusarium graminearum cultivado in vitro. En otro trabajo se concluyó que la biofumigación redujo significativamente la cantidad de esclerocios de Sclerotium rolfsii. Los resultados obtenidos en varios ensayos mostraron que la técnica de biocontrol con el hongo antagonista Trichoderma spp. puede ser considerada compatible con la biofumigación con $B$. juncea. Además, el crecimiento del hongo benéfico Trichoderma spp. no fue afectado por la biofumigación con $B$. juncea. En otro ensayo se observó que la biofumigación con dosis bajas de $B$. juncea no inhibió el crecimiento in vitro de colonias de Azospirillum brasilense, bacteria rizosférica promotora del crecimiento vegetal. Con respecto al efecto de la biofumigación sobre las malezas, se observó reducción del crecimiento de varias especies de malezas: Anoda cristata (malva cimarrona), Picris echiodes, Portulaca oleracea (verdolaga), Digitaria sanguinalis (pasto de cuaresma) y Taraxacum officinalis (diente de león). También se determinó que la biofumigación con SANTA CATALINA UNLP incrementó la densidad de nematodos de vida libre y redujo la densidad de nematodos parásitos de plantas.

Palabras clave: biofumigación; Brassica juncea; biocontrol; manejo integrado de plagas; mejoramiento vegetal 
Obtaining the first Argentine variety of indian mustard: SANTA CATALINA UNLP, for use as biofumigant

Abstract. In recent decades, the greater awareness of society about the harmful effects of agrochemicals on health and the environment, has generated changes in some horticultural and flower farms in the green belt of Buenos Aires, which have incorporated other strategies to control pests, friendly with the environment, and reduced the use of synthetic pesticides. In this context, biofumigation with indian mustard (Brassica juncea L. Czerniak) is presented as an effective biological strategy for the control of soil pests, which could be part of an agroecological pest management program. In our country there are use experiences of biofumigation practices with local resources (mainly, harvest residues of horticultural Brassicaceae species) but there were no cultivar developments for biofumigant purposes. For this reason, a mass selection process was carried out and the biofumigant variety SANTA CATALINA UNLP was obtained, which is the first cultivar of indian mustard registered in the national registry of cultivars (Registro Nacional de Cultivares, INASE).

The SANTA CATALINA UNLP indian mustard variety was developed for use as a biofumigant for soils and substrates in horticultural and floricultural productions. Given that the selection process was carried out in Llavallol town (Buenos Aires Province), this cultivar is adapted to the environmental conditions of the green belt of Buenos Aires. Numerous investigations carried out during the cultivar selection period have demonstrated the efficacy of biofumigation with this variety to suppress some species of phytopathogenic fungi, to reduce populations of phytophagous nematodes and to inhibit the emergence and growth of some weeds. Biofumigation with indian mustard was also shown not to affect the antagonistic fungus Trichoderma spp. nor to the free-living soil nematodes (beneficial).

The SANTA CATALINA UNLP variety was developed at the Instituto Fitotécnico de Santa Catalina, a facility at Facultad de Ciencias Agrarias y Forestales, Universidad Nacional de La Plata. From 2009 to 2016, mass selection was made on a mixed population from a batch of indian mustard seeds that entered the country from India, to be marketed as a condiment. The plants with the highest aerial biomass and with the lowest incidence and severity of black rot or V-spot (Xanthomonas campestris pv. campestris) were selected. At the same time, tests were carried out to evaluate its capacity as a biofumigant for the biocontrol of agricultural 
Innovación y Desarrollo Tecnológico y Social (2021) 3 (1): 1- 23

pests. In June 2017 it was multiplied, verifying the stability of the variety. In several tests it was observed that biofumigation with indian mustard had a suppressing effect on the growth in vitro of Fusarium graminearum. In another work, it was concluded that biofumigation significantly reduced the amount of sclerotia of Sclerotium rolfsii. The results obtained in several trials showed that the biocontrol technique with the Trichoderma spp. antagonist fungus can be considered compatible with $B$. juncea biofumigation. Thus, the growth of the Trichoderma spp. beneficial fungus it was not affected by biofumigation with $B$. juncea. In another trial, it was observed that biofumigation with low doses of $B$. juncea did not inhibit the in vitro growth of colonies of Azospirillum brasilense, a plant growth-promoting rhizobacteria. Regarding the effect of biofumigation on weeds, a reduction in the growth of several weed species was observed: Anoda cristata, Picris echiodes, Portulaca oleracea, Digitaria sanguinalis and Taraxacum officinalis. It was also determined that biofumigation with SANTA CATALINA UNLP increased the density of free-living nematodes and reduced the density of plant parasitic nematodes.

Key words: biofumigation; Brassica juncea; biocontrol; integrated pest management; plant breeding

\section{Novedad u originalidad local en el conocimiento}

La desinfección de suelos es una práctica habitual en el cinturón verde de Buenos Aires, tanto en las producciones hortícolas como en las florícolas. Durante muchos años, la fumigación de suelos y sustratos con bromuro de metilo fue una práctica generalizada y de buenos resultados, para controlar malezas y otras plagas en las primeras etapas de los cultivos hortícolas, especialmente en producciones bajo invernáculo. Debido a la prohibición del uso del bromuro de metilo, a causa de su efecto nocivo sobre la capa de ozono (Methyl Bromide Technical Options 
Innovación y Desarrollo Tecnológico y Social (2021) 3 (1): 1- 23

Committee, 1994), se lo sustituyó gradualmente por algunos pesticidas sintéticos y, en menor medida, por otros métodos, como la solarización y la inyección de vapor de agua (Biaggi et al., 2011; Pizano, 2014). Es importante continuar con la búsqueda de nuevas alternativas para el manejo integrado de plagas, que sean eficaces y amigables con el medio ambiente.

Una de esas alternativas es la biofumigación con especies de la Familia Brassicaceae (brasicáceas), que puede definirse como el control de plagas y patógenos edáficos por medio de la incorporación en el suelo de ciertas especies de brasicáceas, que liberan diferentes tipos de isotiocianatos con actividad biocida, como resultado de la hidrólisis de los glucosinolatos contenidos en sus tejidos (Kirkegaard et al., 1993). Entre las especies de brasicáceas más estudiadas como biofumigantes se encuentra Brassica juncea L. Czerniak (mostaza india). El desarrollo de variedades de esta especie, adaptadas a las condiciones locales, representa un aporte al manejo agroecológico de plagas del suelo. En nuestro país hay registros de prácticas de biofumigación con recursos locales (principalmente, restos de cosecha de especies hortícolas brasicáceas), pero no existían desarrollos de cultivares con propósitos biofumigantes. Por este motivo, se realizó un proceso de selección masal y se obtuvo la variedad biofumigante SANTA CATALINA UNLP, que es el primer cultivar de mostaza india inscripto en el Registro Nacional de Cultivares del Instituto Nacional de Semillas (INASE). 
Innovación y Desarrollo Tecnológico y Social (2021) 3 (1): 1- 23

\section{Grado de relevancia}

El control de plagas agrícolas mediante tácticas biológicas, entre las que se encuentra la biofumigación con mostaza india, es una de las herramientas del manejo integrado de plagas. En las últimas décadas, la mayor concientización de la sociedad sobre los efectos nocivos de los agroquímicos sobre la salud y el medio ambiente ha generado cambios en algunos establecimientos hortícolas y florícolas, que han incorporado otras estrategias de control de plagas amigables con el medio ambiente, reduciendo el uso de plaguicidas sintéticos o sustituyéndolos completamente, como en el caso de los productores orgánicos. Este cambio también fue debido, en nuestro país, al elevado costo de los pesticidas, que en muchos casos reduce notablemente los márgenes económicos o hace inviables los cultivos. Desde el punto de vista agroecológico, la práctica de la biofumigación con mostaza india presenta ventajas con respecto a los fumigantes de suelo sintéticos, como el metam sodio y el dazomet: es inocua para el agricultor que la ejecuta, no contamina el suelo ni deja residuos tóxicos para los cultivos que se implantan posteriormente; además, la incorporación de enmiendas biofumigantes mejora la estructura y la penetración del agua, aumenta el contenido de materia orgánica del suelo y reduce el encostramiento (Pung et al., 2008). Por otro lado, la biofumigación con mostaza india es una práctica de bajo costo económico y de fácil implementación por parte de los agricultores. 


\section{Grado de pertinencia}

La variedad de mostaza india SANTA CATALINA UNLP fue desarrollada para su utilización como biofumigante de suelos y sustratos en producciones hortícolas y florícolas. Dado que el proceso de selección se realizó en la localidad de Llavallol, conurbano bonaerense, este cultivar se adapta a las condiciones medioambientales del cinturón verde de Buenos Aires.

Numerosas investigaciones realizadas durante el período de selección del cultivar han demostrado la eficacia de la biofumigación con mostaza india para suprimir algunas especies de hongos fitopatógenos, reducir poblaciones de nematodos fitófagos y para inhibir la emergencia y el crecimiento de algunas malezas. También se demostró que la biofumigación con mostaza india no afecta al hongo antagonista Trichoderma spp. ni a los nematodos de vida libre del suelo (benéficos) (Chorzempa et al., 2019; D'Amico et al., 2019; Perniola et. al., 2011 a; 2011 b; 2012; 2014; 2015; 2016 a; 2016 b; 2018; 2019).

La biofumigación con mostaza india debería formar parte de un programa de manejo integrado de plagas, en combinación con otras tácticas de control; su utilización por sí sola no generará, a mediano y largo plazo, los mismos resultados que los obtenidos cuando se la integra con otras estrategias culturales y biológicas. Requiere una mínima capacitación por parte de los agricultores para poder obtener los resultados deseados. 
Innovación y Desarrollo Tecnológico y Social (2021) 3 (1): 1- 23

\section{Grado de demanda}

Los agentes demandantes del cultivar SANTA CATALINA UNLP estarían representados por los productores hortícolas y florícolas del cinturón verde de Buenos Aires, que requieren metodologías alternativas a las convencionales para la fumigación de suelos y sustratos, ya sea por búsqueda de tácticas agroecológicas, por requerimiento legal en el caso de las producciones orgánicas certificadas o por conveniencia económica en comparación con el uso de pesticidas sintéticos.

En otros casos, por exigencias relacionadas con políticas medioambientales, algunos productores requieren adquirir este tipo de tecnologías para adecuarse a las normativas vigentes. Este es el caso de los quinteros del Parque Pereyra Iraola (PPI), en el cinturón hortícola de Buenos Aires, que realizaron una conversión productiva centrada en los principios de la agroecología, para poder permanecer en las tierras que ocupaban legalmente desde la expropiación del PPI por el gobierno provincial. A partir de 2004, los productores del PPI iniciaron las primeras experiencias en biofumigación con especies brasicáceas para el control de fitonematodos, con resultados alentadores (Bongiorno et al., 2009).

\section{Desarrollo del producto}

La variedad de mostaza india denominada SANTA CATALINA UNLP fue desarrollada en el Instituto Fitotécnico de Santa Catalina, dependencia de la Facultad de Ciencias Agrarias y Forestales de la Universidad Nacional de La Plata, situado en la localidad 
Innovación y Desarrollo Tecnológico y Social (2021) 3 (1): 1- 23

de Llavallol, provincia de Buenos Aires (3447'21,5"S; 58²6'48,5"O). Desde el año 2009 hasta el 2016 se realizó selección masal sobre una población mixta proveniente de un lote de semillas de mostaza india, que ingresó al país procedente de la India para ser comercializado como condimento. Se seleccionaron las plantas con mayor biomasa aérea y con menor incidencia y severidad de podredumbre negra o mancha en V (Xanthomonas campestris pv. campestris). Paralelamente se realizaron ensayos para evaluar su capacidad como biofumigante para el biocontrol de plagas agrícolas (Chorzempa et al., 2019; D’Amico et al., 2019; Perniola et. al., 2011 a; 2011 b; 2012; 2014; 2015; 2016 a; 2016 b; 2018; 2019). En junio de 2017 fue multiplicada, verificándose la estabilidad de la variedad.

\section{Propósito agronómico de la variedad SANTA CATALINA UNLP}

La variedad SANTA CATALINA UNLP fue seleccionada para su utilización como biofumigante. La biofumigación es el control de plagas y patógenos edáficos por medio de la incorporación en el suelo de ciertas especies de brasicáceas, que liberan diferentes tipos de isotiocianatos con actividad biocida, como resultado de la hidrólisis de los glucosinolatos contenidos en sus tejidos (Kirkegaard et al., 1993). Durante el proceso de biofumigación, los glucosinolatos presentes en los tejidos de las brasicáceas (Kjaer, 1976), se hidrolizan por la acción de la enzima mirosinasa produciendo diferentes tipos de isotiocianatos, con variable grado de toxicidad frente a diversos organismos (Molina-Vargas \& Bentura-Castellanos, 2009). Según 
Innovación y Desarrollo Tecnológico y Social (2021) 3 (1): 1- 23

Gimsing y Kirkegaard (2009), la efectividad de la biofumigación se basa en lograr la máxima hidrólisis de los glucosinolatos presentes en el tejido vegetal, para generar altas concentraciones de isotiocianatos en el suelo después de su incorporación; esto es favorecido por una gran disrupción celular, la adición de agua y alta temperatura del suelo.

La biofumigación puede inhibir la emergencia y crecimiento de malezas (Norsworthy et al., 2005; Anderson et al., 2008; Boydston et al., 2008; 2011; Perniola et al., 2011 a; Webber et al., 2012; Perniola et al., 2016 a). También se ha demostrado la eficacia de esta técnica para reducir las poblaciones de nematodos (Pattison et al., 2003; Mitidieri et al., 2009) y hongos fitopatógenos (Kirkegaard et al., 1996; Iriarte et al., 2011; Perniola et al., 2012; 2014; Lafi et al., 2017). La efectividad del tratamiento biofumigante varía según el estadio fenológico de la mostaza. La mayoría de los estudios realizados en el tema, citan un buen efecto biofumigante en el período comprendido entre plena floración y fin de fructificación. La variedad SANTA CATALINA UNLP ha sido evaluada, con resultados satisfactorios, en el estadio de fructificación.

Resultados de los ensayos experimentales realizados para determinar la capacidad biofumigante de la variedad

En varios ensayos se observó que la biofumigación con mostaza india tuvo efecto supresor sobre el crecimiento de Fusarium graminearum cultivado in vitro (Perniola 
Innovación y Desarrollo Tecnológico y Social (2021) 3 (1): 1- 23

et al., 2011 b; 2012; 2014; 2015). En otro trabajo se concluyó que la biofumigación con 2,26 kg. $\mathrm{m}^{-2}$ de B. juncea redujo significativamente la cantidad de esclerocios de Sclerotium rolfsii (Chorzempa et al., 2019). Los resultados obtenidos en varios ensayos mostraron que la técnica de biocontrol con el hongo antagonista Trichoderma spp. puede ser considerada compatible con la biofumigación con $B$. juncea (Chorzempa et al., 2019; Perniola et al., 2014; 2015; 2016 b). El crecimiento del hongo benéfico Trichoderma spp. no fue afectado por la biofumigación con B. juncea. Además, la utilización combinada de Trichoderma spp. y la biofumigación con $B$. juncea, tuvo un efecto sinérgico sobre el control del crecimiento de F. graminearum (Perniola et al., 2014; 2015). En otro ensayo se observó que la biofumigación con dosis bajas de B. juncea no inhibió el crecimiento in vitro de colonias de Azospirillum brasilense, bacteria rizosférica promotora del crecimiento vegetal (Perniola et al., 2016 b).

Con respecto al efecto de la biofumigación con B. juncea sobre las malezas, se observó reducción del crecimiento de malezas (Perniola et al., 2011 a; 2016 a; 2018; 2019). En un ensayo in vitro se observó inhibición significativa de la germinación de las malezas Anoda cristata (malva cimarrona), Picris echiodes y Portulaca oleracea (verdolaga) (Perniola et al., 2016 a). En otro ensayo realizado en macetones, se observó que la dosis de 2,5 kg.m-2 de materia fresca redujo el número total de individuos de arvenses mono y dicotiledóneas, en particular de Digitaria sanguinalis (pasto de cuaresma), P. oleracea (verdolaga) y Taraxacum officinalis (diente de león) 
Innovación y Desarrollo Tecnológico y Social (2021) 3 (1): 1- 23

(Perniola et al., 2019). En condiciones de campo, la biofumigación con 2,26 kg.m ${ }^{-2}$ de materia fresca provocó una disminución del 83,4 \% en el número de individuos de verdolaga y del 90,9 \% en el peso seco total, con respecto al control (Perniola et al., 2018).

Con respecto al efecto de la biofumigación con $B$. juncea sobre los nematodos, la biofumigación con $2 \mathrm{~kg} \cdot \mathrm{m}^{-2}$ de materia fresca incrementó la densidad de nematodos de vida libre (D’Amico et al., 2019) y redujo la densidad de nematodos parásitos de plantas (D’Amico et al., inédito, 2020).

En un ensayo realizado en condiciones de campo se demostró que la biofumigación con 2,26 kg. $\mathrm{m}^{-2}$ de B. juncea incrementó la velocidad de emergencia y la cantidad de plántulas de lechuga (Lactuca sativa), sembrada después de practicar la biofumigación (Chorzempa et al., 2019). Es necesario continuar con la realización de ensayos experimentales, para determinar el espectro total de control de plagas de la variedad SANTA CATALINA UNLP.

\section{Características fenotípicas de la variedad SANTA CATALINA UNLP}

En la Figura 1 se observan detalles de la planta en distintos estadios fenológicos y en la Figura 2 una vista general de una parcela en plena floración. En la Tabla 1 se detallan los valores medios de algunos caracteres medidos en la variedad SANTA CATALINA UNLP (Unión Internacional para la Protección de las Obtenciones Vegetales, 2020). 


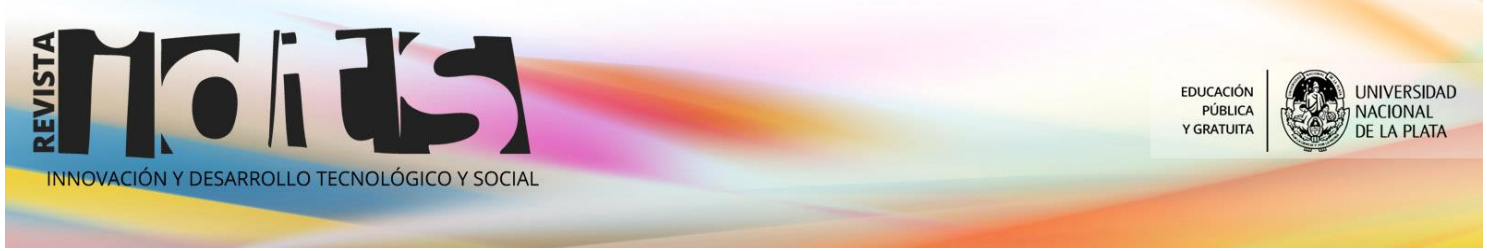

Innovación y Desarrollo Tecnológico y Social (2021) 3 (1): 1- 23

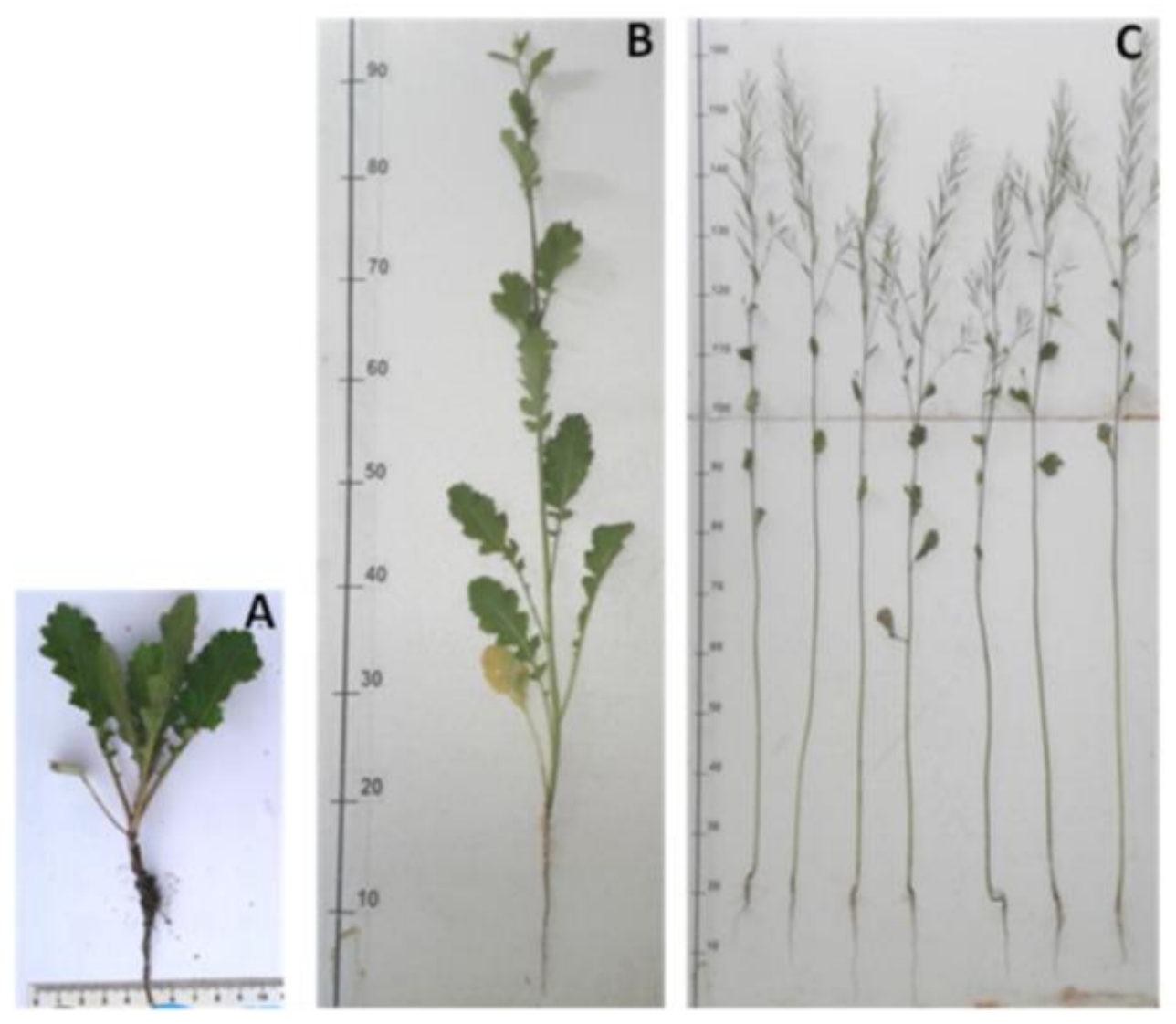

Figura 1. Plantas de la variedad SANTA CATALINA UNLP en distintos estadios fenológicos. A: un entrenudo alargado, 60 días desde la siembra; B: botones florales libres, al mismo nivel que las hojas más jóvenes, 73 días desde la siembra; C: casi todas las silicuas han alcanzado su tamaño definitivo, 114 días desde la siembra.

Figure 1. Plants of the SANTA CATALINA UNLP variety in different phenological stages. A: an elongated internode, 60 days from sowing; B: free flower buds, at the same level as the youngest leaves, 73 days from sowing; C: almost all siliques have reached their final size, 114 days from sowing. 
Tabla 1. Valores medios y desvíos estándar de algunos caracteres medidos en la variedad SANTA CATALINA UNLP.

Table 1. Average values and standard deviations of some characters measured in the SANTA CATALINA UNLP variety.

\begin{tabular}{|c|c|c|c|}
\hline \multirow[b]{2}{*}{ Carácter } & \multirow{2}{*}{$\begin{array}{l}\text { Estadio } \\
\text { fenológico de la } \\
\text { medición (clave) }^{1}\end{array}$} & \multicolumn{2}{|c|}{ Valor medido } \\
\hline & & Media & $\begin{array}{l}\text { Desvío } \\
\text { estándar }\end{array}$ \\
\hline Cotiledón: longitud (mm) & 10 & 5,77 & 0,54 \\
\hline Cotiledón: anchura (mm) & 10 & 10,98 & 0,87 \\
\hline Hoja: longitud (cm) & 19 & 17,12 & 2,11 \\
\hline Hoja: anchura (cm) & 19 & 5,35 & 0,68 \\
\hline Hoja: longitud del pecíolo (cm) & 19 & 4,84 & 1,11 \\
\hline Hoja: anchura del pecíolo (cm) & 19 & 0,50 & 0,08 \\
\hline Limbo: longitud del lóbulo terminal (cm) & 19 & 9,00 & 0,92 \\
\hline Limbo: anchura del lóbulo terminal (cm) & 19 & 5,35 & 0,68 \\
\hline Limbo: número de lóbulos laterales & 19 & 4,33 & 1,09 \\
\hline Limbo: pubescencia en envés ${ }^{2}$ & 19 & 1,23 & 1,38 \\
\hline Limbo: intensidad del color verde ${ }^{3}$ & 19 & 5,43 & 0,53 \\
\hline Limbo: densidad de las incisiones del borde ${ }^{4}$ & 19 & 1,85 & 0,27 \\
\hline Planta: longitud (m) & $70-79$ & 1,36 & 0,08 \\
\hline Silicua: longitud (cm) & $70-79$ & 3,44 & 0,29 \\
\hline Silicua: anchura $(\mathrm{cm})$ & $70-79$ & 0,38 & 0,04 \\
\hline Silicua: longitud de la punta $(\mathrm{cm})$ & $70-79$ & 0,93 & 0,13 \\
\hline Silicua: longitud del pedúnculo (cm) & $70-79$ & 1,21 & 0,13 \\
\hline Contenido de glucosinolatos ${ }^{5}\left(\mathrm{mg} \cdot \mathrm{kg}^{-1}\right)$ & 69 & 939,50 & 9,19 \\
\hline
\end{tabular}

${ }^{1}$ Según Meier (2001) y UPOV (2020): clave 10: cotiledones totalmente desplegados; clave 19: 9 o más hojas desplegadas; clave 69: casi todas las silicuas han alcanzado su tamaño definitivo; clave 70: semilla verde, se está llenando la cavidad de la silicua; clave 79: maduración plena, casi todas las silicuas están maduras (semillas oscuras y duras). 
Innovación y Desarrollo Tecnológico y Social (2021) 3 (1): 1- 23

${ }^{2}$ Número de pelos por centímetro lineal, contabilizados en el envés de la nervadura central de la quinta hoja. La medición se realiza en el envés de la nervadura central, porque es la región del limbo que concentra la mayor cantidad de pelos.

${ }^{3}$ Contenido relativo de clorofila en la quinta hoja, medido con un medidor Hansatech CL-01.

${ }^{4}$ Cantidad de incisiones por centímetro lineal contabilizadas en la parte distal de la quinta hoja.

${ }^{5}$ Contenido de glucosinolatos totales en la parte aérea de la planta. Se expresa en miligramos de glucosinolatos por kilogramo de materia fresca de mostaza. Método de evaluación: indirecta, por medición de la actividad enzimática de la mirosinasa mediante titulación de homogenatos de biomasa con $\mathrm{NaOH}$.

${ }^{1}$ According to Meier (2001) and UPOV (2020): key 10: fully expanded cotyledons; key 19: 9 or more leaves unfolded; key 69: almost all siliques have reached their final size; key 70: green seed, the cavity of the silique is filling; key 79: full maturation, almost all siliques are mature (dark and hard seeds).

${ }^{2}$ Number of hairs per linear centimeter, counted on the underside of the midrib of the fifth leaf. The measurement is made on the underside of the midrib, because it is the region of the limbus that concentrates the greatest amount of hairs.

${ }^{3}$ Relative chlorophyll content in the fifth leaf, measured with a Hansatech CL-01 meter.

${ }^{4}$ Number of incisions per linear centimeter counted in the distal part of the fifth leaf.

${ }^{5}$ Content of total glucosinolates in the aerial part of the plant. It is expressed in milligrams of glucosinolates per kilogram of fresh mustard matter. Evaluation method: indirect, by measuring the enzymatic activity of myrosinase by titration of biomass homogenates with $\mathrm{NaOH}$.

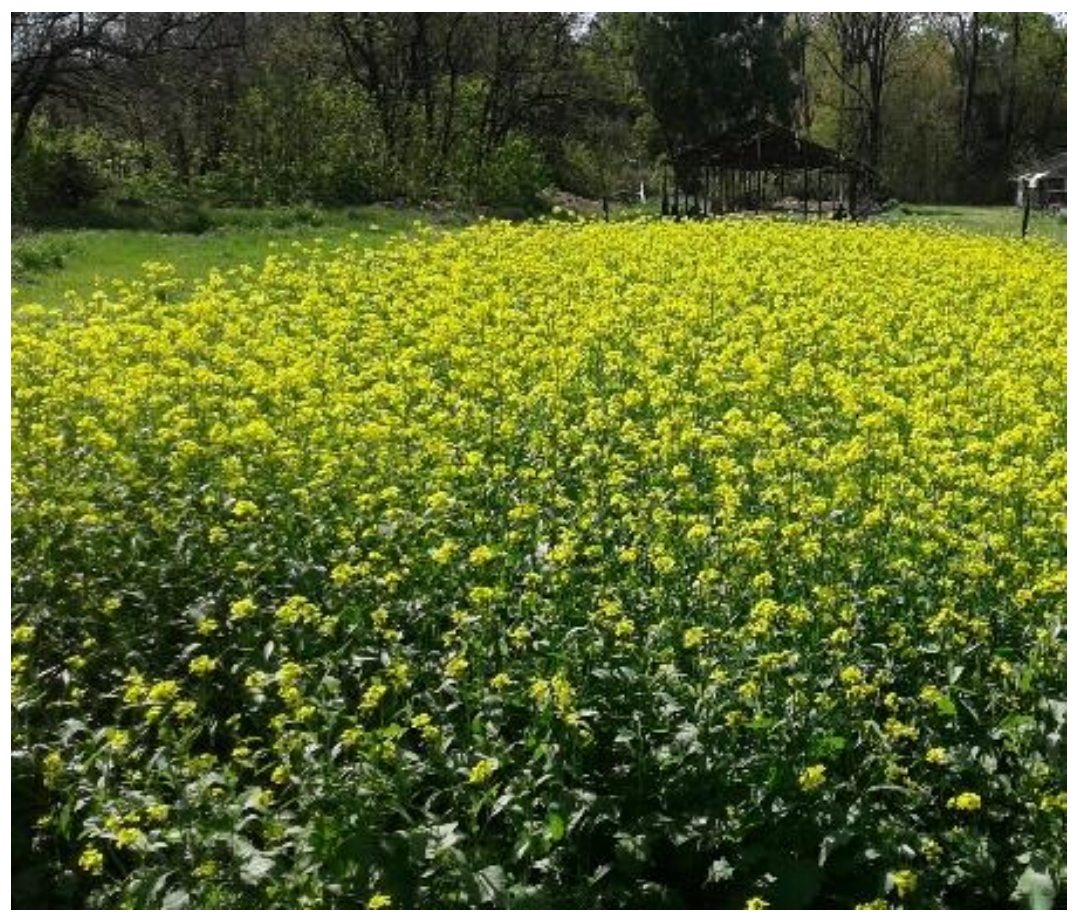

Figura 2. Vista general de una parcela de la variedad SANTA CATALINA UNLP en plena

floración. Jardín Experimental del Instituto Fitotécnico de Santa Catalina, Llavallol, Buenos Aires. 
Innovación y Desarrollo Tecnológico y Social (2021) 3 (1): 1- 23

Figure 2 (previous page). General view of a plot of SANTA CATALINA UNLP variety in full bloom. Experimental Garden of Instituto Fitotécnico de Santa Catalina, Llavallol, Buenos Aires.

Información sobre el patentamiento/registro de la innovación o desarrollo

La variedad de mostaza india SANTA CATALINA UNLP fue inscripta en el Registro Nacional de Cultivares del Instituto Nacional de Semillas, Ministerio de Agricultura, Ganadería y Pesca, el 27 de octubre de 2020, por Resolución № RESOL-2020-346APN-INASE\#MAGYP, con Nº de Registro: 19459.

\section{Financiamiento}

Proyecto de Investigación y Desarrollo: Alternativas de control biológico en programas de manejo integrado de plagas en cultivos agrícolas. Código de proyecto: A311, subsidiado por la Universidad Nacional de La Plata.

\section{Referencias bibliográficas}

Anderson, D., Masiunas, J.B., Bossu, S. y Kushad, M. (2008). Mustards as biofumigants: current status and future prospects. North Central Weed Sci. Soc. Abstr., 63, 172. [CD-ROM computer file]. North Central Weed Sci. Soc., Champaign, Illinois, USA. 
Biaggi, C., Mondzak, F. y Valeiro, A. (2011). Problemas ambientales, oportunidades de desarrollo: la experiencia del Proyecto INTA Prozono. Ediciones INTA. Tucumán. 118 pp.

Bongiorno, M., Larrosa, C., Maidana, A., Arenas, M., Cruz, Y., López, R., Gianuzzi, L. y Cap, G. (2009). Biofumigación con recursos locales: el caso de la producción hortícola de los quinteros del Parque Pereyra Iraola. LEISA Revista de Agroecología, 25(4), 25-28. Recuperado de: http://www.leisaal.org/web/images/stories/revistapdf/vol25n4.pdf

Boydston, R.A., Anderson, T. y Vaughn, S.F. (2008). Mustard (Sinapis alba) seed meal suppresses weeds in container-grown ornamentals. HortScience, 43(3), 800803.

Boydston, R.A., Morra, M.J., Borek, V., Clayton, L. y Vaughn, S.F. (2011). Onion and weed response to mustard (Sinapis alba) seed meal. Weed Science, 59, 546-552.

Chorzempa, S.E., Perniola, O.S., Subelza, L.F., Barca, H.J., Sánchez, S.E., Rodas Alonso, R., Molina, M. del C. y Astiz Gassó, M.M. (2019). Biofumigación con Brassica juncea. Efecto sobre Trichoderma harzianum, Sclerotium rolfsii y Lactuca sativa. XXXVII Jornadas Argentinas de Botánica, San Miguel de Tucumán, 9 al 13 de Septiembre de 2019. Bol. Soc. Argent. Bot., 54 (Supl.), 136-137. Recuperado de: https://botanicaargentina.org.ar/wp-content/uploads/2019/09/JornadasArgentinas-de-Bot\%C3\%A1nica-2019-1-1.pdf 
D’Amico, M., Perniola, O.S., Chorzempa, S.E., Cap, G.B., Castaldo, V., Olaechea, B., Sánchez, E.C., Parisi, N.E., Odda, M., Astiz Gassó, M.M., Salas, A., Arcuri, J.E. y Molina, M. del C. (2019). Biofumigación con Mostaza parda (Brassica juncea L. Czern.). Efecto sobre la Nematofauna del Suelo. Primer Congreso Argentino de Agroecología, Mendoza. 18 al 20 de septiembre de 2019. Libro de resúmenes: 466-469. Recuperado de:

https://bdigital.uncu.edu.ar/objetos digitales/14315/libroresumencongresoargentinoagroecologia.pdf

D'Amico, M., Perniola, O.S., Chorzempa, S.E., Cap, G.B., Castaldo, V., Olaechea, B., Sánchez, E.C., Parisi, N.E., Odda, M., Astiz Gassó, M.M., Salas, A., Arcuri, J.E. y Molina, M. del C. (2020). Biofumigación con Mostaza parda (Brassica juncea L. Czern.). Efecto sobre nematodos parásitos y de vida libre del suelo. Manuscrito inédito.

Gimsing, A.L. y Kirkegaard, J.A. (2009). Glucosinolates and biofumigation: fate of glucosinolates and their hydrolysis products in soil. Phytochem Rev., 8, 299-310. Iriarte, L.E., Sosa, M.C. y Reybet, G.E. (2011). Efecto de la biofumigación con repollo sobre el control de Fusarium oxysporum en suelo. RIA, 37(3), 231-237.

Kirkegaard, J.A., Gardner, P.A., Desmarchelier, J.M. y Angus, J.F. (1993). Biofumigation using Brassica species to control pests and diseases in horticulture and agriculture. Proceedings of 9th Australian Research Assembly on Brassicas. Wagga Wagga, Australia. pp. 77-82. 
Innovación y Desarrollo Tecnológico y Social (2021) 3 (1): 1- 23

Kirkegaard, J.A., Wong, P.T.W. y Desmarchelier, J.M. (1996). In vitro supression of fungal root pathogens of cereals by Brassica tissues. Plant Pathol., 45, 593-603. Kjaer, A. (1976). Glucosinolates in cruciferae. En: The Biology and Chemistry of the Cruciferae. Academic Press, London. pp. 207-219.

Lafi, J.G., Tarquini, A.M., Sanz Pérez, M. y Puglia, M.C. (2017). Susceptibilidad in vitro de Fusarium spp, patógenas en tomate, a biofumigación con Brassicáceas. Procede de $4^{\circ}$ Congreso Argentino de Fitopatología. Mendoza, Argentina. pp. 363.

Meier, U. (2001). Growth stages of mono-and dicotyledonous plants. BBCH Monograph. Federal Biological Research Centre for Agriculture and Forestry. 158 pp. Recuperado de: https://www.politicheagricole.it/flex/AppData/WebLive/Agrometeo/MIEPFY800 /BBCHengl2001.pdf

Methyl Bromide Technical Options Committee (MBTOC). (1994). Report of the Methyl Bromide Technical Options Committee. Montreal Protocol on substances that deplete the ozone layer. UNEP, Kenya. pp. 304.

Mitidieri, M., Brambilla, V., Saliva, V., Piris, E., Piris, M., Celié, R., Pereyra, C., Del Pardo, K., Chaves, E. y González, J. (2009). Efecto de distintas secuencias de tratamientos de biofumigación sobre parámetros fisicoquímicos y biológicos del suelo, el rendimiento y la salinidad de cultivos de tomate y lechuga bajo cubierta. Horticultura Argentina, 28(67), 5-17. 
Innovación y Desarrollo Tecnológico y Social (2021) 3 (1): 1- 23

Molina-Vargas, L.F. y Bentura-Castellanos, J.U. (2009). Efecto inhibitorio in vitro de cinco isotiocianatos sobre Rhizoctonia solani Kühn AG-3. Revista de Investigación Agraria y Ambiental, 37-40.

Norsworthy, J.K., Brandenberger, L., Burgos, N.R. y Riley, M. (2005). Weed suppression in Vigna unguiculata with a spring-seeded brassicaceae green manure. Crop Protection, 24(5), 441-447.

Pattison, T., Martin, T., Akiew, S., Versteeg, C. y Kirkegaard, J. (2003). Can Brassicas be used to manage root-knot nematode in tropical vegetal production? Australasian Nematology Newsletter, 14(2), 16-19.

Perniola, O.S., Chorzempa, S.E., Staltari, S., Rodriguez, H. y Molina, M. del C. (2011 a). Incidencia de la biofumigación sobre el control de malezas. VIII Simposio Nacional de Biotecnología REDBIO Argentina 2011. C.A.B.A., 13 al 15 de Noviembre de 2011. Sección 3-54. Recuperado de: http://www.redbioargentina.org.ar/simposio2011/seccion3.html

Perniola, O.S., Staltari, S., Chorzempa, S.E. y Molina, M. del C. (2011 b). Biofumigación con Brassicáceas: actividad supresora sobre Fusarium graminearum. $2^{\circ}$ Congreso Argentino de Fitopatología. Mar del Plata, Buenos Aires, 1, 2 y 3 de Junio de 2011. Libro de resúmenes, 326. Recuperado de: http://www.aafitopatologos.com.ar/media/secciones/415 desc.pdf

Perniola, O.S., Staltari, S., Chorzempa, S.E. y. Molina, M. del C. (2012). Biofumigación con Brassicáceas: actividad supresora sobre Fusarium graminearum. Rev. Fac. 
Innovación y Desarrollo Tecnológico y Social (2021) 3 (1): 1- 23
Agron.,
$111(1)$
48-53.
Recuperado
de:

http://revista.agro.unlp.edu.ar/index.php/revagro/article/view/71/46

Perniola, O.S., Staltari, S., Chorzempa, S.E., Astiz Gassó, M.M. y Molina, M. del C. (2014). Control biológico de Fusarium graminearum: utilización de Trichoderma spp. y biofumigación con parte aérea de Brassica juncea. Rev. Fac. Cienc. Agrar. UNCuyo, 46(2), 45-56. Recuperado de: http://revista.fca.uncu.edu.ar/images/stories/pdfs/2014-02/Cp04 Perniola.pdf Perniola, O.S., Chorzempa, S.E., Staltari, S., Astiz Gassó, M.M. y Molina, M. del C. (2015). Integración de dos métodos de biocontrol in vitro de Fusarium graminearum. XVII Congreso Internacional y XLII Congreso Nacional de la Sociedad Mexicana de Fitopatología. Ciudad de México, México. 19 al 23 de Julio de 2015. Revista Mexicana de Fitopatología, 33, S128. Recuperado de: http://rmf.smf.org.mx/suplemento/docs/Volumen332015/Suplemento332015F $\underline{\text { ULL.pdf }}$

Perniola, O.S., Chorzempa, S.E., Staltari, S. y Molina, M. del C. (2016 a). Biofumigación in vitro con Brassica juncea y Sinapis alba. Inhibición de la germinación y del crecimiento de plántulas de malezas. Rev. Fac. Agron., 115(1), 91-98. Recuperado de: http://revista.agro.unlp.edu.ar/index.php/revagro/article/view/290/305

Perniola, O.S., Chorzempa, S.E., Staltari, S., Astiz Gassó, M.M., Galian, L.R. y Molina, M. del C. (2016 b). Biofumigación con Brassica juncea L. Czerniak y Sinapis alba L. 
Innovación y Desarrollo Tecnológico y Social (2021) 3 (1): 1- 23

Acción sobre el crecimiento in vitro de Trichoderma spp. y Azospirillum brasilense

Tarrand, Krieg et Döbereiner. 2016. Rev. Protección Veg., 31(1), 57-62.

Recuperado de: $\underline{\text { http://scielo.sld.cu/pdf/rpv/v31n1/rpv08116.pdf }}$

Perniola, O.S., Chorzempa, S.E., Subelza, L.F., Barca, H.J., Sánchez, S.E., Rodas, R., Molina, M. del C. y Astiz Gassó, M.M. (2018). Biofumigación con mostaza parda (Brassica juncea). Efecto sobre la maleza verdolaga (Portulaca oleracea). XVI Jornadas Fitosanitarias Argentinas. Tucumán. 10 al 12 de Octubre de 2018. Revista de Agronomía del Noroeste Argentino 38 (1), 86-87. Recuperado de: http://www.jfa2018.unt.edu.ar/pdf/jfa 2018.pdf

Perniola, O.S., Chorzempa, S.E., Staltari, S. y Molina, M. del C. (2019). Biofumigación con Brassica juncea: efecto sobre la flora arvense. Rev. Fac. Agron., 118(1), 2535. Recuperado de:

http://revista.agro.unlp.edu.ar/index.php/revagro/article/view/1130/1664

Pizano, M. (2014). Eliminación del bromuro de metilo en países en vías de desarrollo. Una historia de éxito y sus retos. Programa de las Naciones Unidas para el Medio Ambiente. París. 60 pp.

Pung, H., Cross, S. y Patten, D. (2008). The use of biofumigant green manure crops for soil-borne disease management in Tasmania. Proceedings of Third International Biofumigation Symposium. Canberra, Australia. pp. 26.

UPOV (Unión Internacional para la Protección de las Obtenciones Vegetales). (2020). Proyecto mostaza de sarepta, mostaza india (Brassica juncea L. Czern.). 


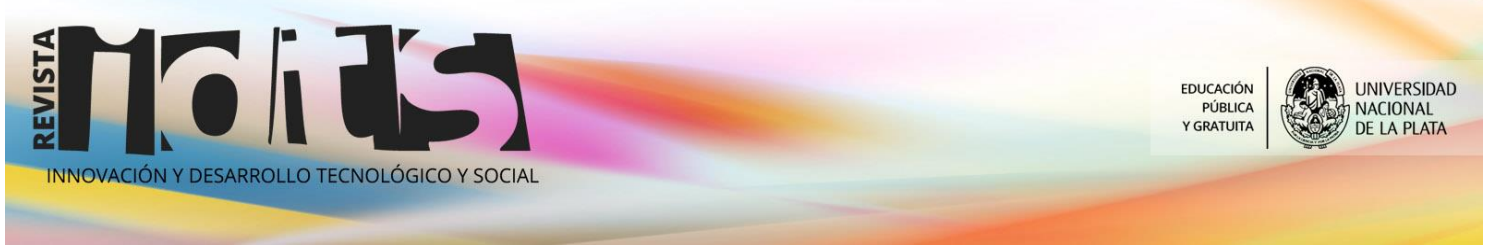

Innovación y Desarrollo Tecnológico y Social (2021) 3 (1): 1- 23

Directrices para la ejecución del examen de la distinción, la homogeneidad y la estabilidad. Ginebra. 33 pp. Recuperado de: https://www.upov.int/edocs/mdocs/upov/es/tc 56/tg brass jun proj 8.pdf

Webber, C.L., Shrefler, J.W. y Brandenberger, L.P. (2012). Organic Weed Control. In: Herbicides - Environmental Impact Studies and Management Approaches. Alvarez-Fernandez R. (Ed.). Publisher: InTech. Rijeka, Croatia. pp. 185-198. 